Acta Cryst. (1954). 7, 545

\title{
The Geometrical Basis of Crystal Chemistry. Part 2
}

\author{
By A. F. WeLLS \\ Imperial Chemical Industries Limited (Dyestuffs Division), Hexagon House, Manchester 9, England
}

(Received 1 January 1954 and in revised form 1 April 1954)

A procedure for the systematic derivation of three-dimensional 4-connected nets is outlined and applied to nets with 2,3 and 4 points in the repeat unit. The relevance to crystal chemistry of nets containing loops (digons) is noted. Some of the simplest open packings of tetrahedra are indicated, and a number of more complex nets (4-, 5-, 6-, 8- and 12-connected) with cubic symmetry are listed.

\section{Introduction}

In the three-dimensional 3-connected nets considered in Part 1 (Wells, 1954) the number $(N)$ of points in the repeat unit must be even, and the minimum value of $N$ is 4 . In three-dimensional 4-connected nets the minimum value of $N$ is 2 and all higher integral values are possible. We shall derive systematically the nets with 2, 3 and 4 points in the repeat unit, but we shall also refer to some more complex 4-connected nets which are of interest in connection with certain crystal structures.

In nets in which four or more links meet at each point there is the possibility that some or all of the links outline polyhedra:

(a) there are discrete polyhedra,

(b) all space is divided into polyhedra of one or more kinds.

If we proceeded with the derivation of nets to a sufficient degree of complexity we should therefore include examples of the partitioning of space into poly. hedra. Reference will be made to relevant aspects of this problem.

\section{Derivation of three-dimensional 4-connected nets}

These may be derived by joining up some or all of the points in planar nets to points in adjacent layers so that all the points become 4-connected. Planar nets of four types have to be considered:

(a) 3-connected nets: Equal numbers of points in each layer must be joined to points in adjacent layers above and below.

(b) Mixed 3- and 4-connected nets: Here the 3connected points are to be joined either upwards or downwards to 3-connected points of adjacent layers. The number of 3 -connected points in the repeat unit must clearly be even.

(c) Mixed 2- and 4-connected nets: Each of the 2connected points must form two additional links to points in adjacent layers.

(d) Mixed 2-, 3- and 4-connected nets: The 2- and 3-connected points form additional links as in (b) and (c).

The simpler 3-connected plane nets have been listed and illustrated in Part 1 (Table 1 and Fig. 1). The 4-connected plane nets required in $(c)$ are derived by finding the ways in which a plane can be divided into polygons. If $\varphi_{n}$ is the fraction of the total number of polygons which are $n$-gons then
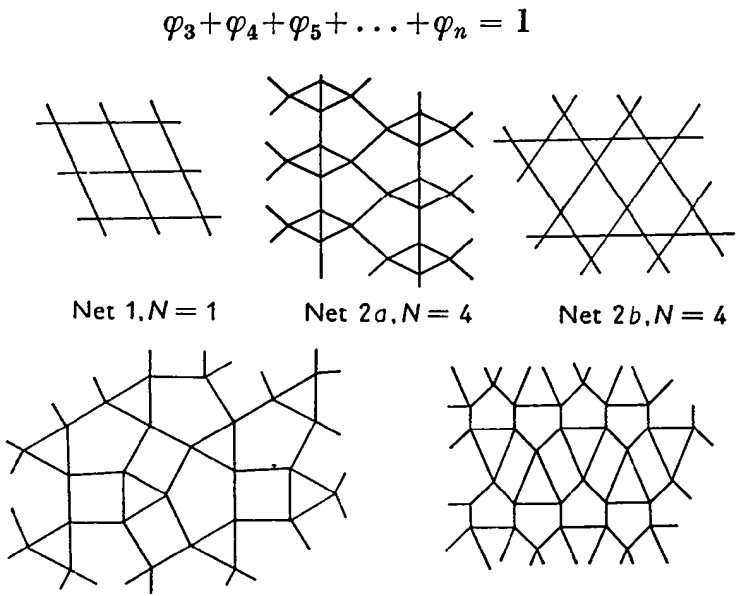

Net $3, N=9$

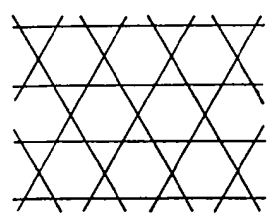

Net $3 . N=6$

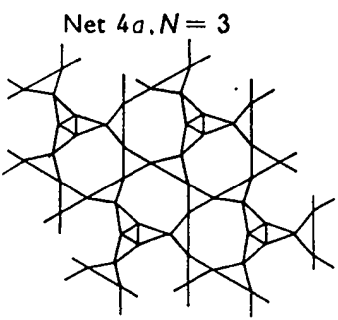

Net $5, N=12$

Fig. 1. Some 4-connected plane nets. 
and

$$
3 \varphi_{3}+4 \varphi_{4}+5 \varphi_{5}+\ldots+n \varphi_{n}=4
$$

The first six solutions of these equations are listed in Table 1, and the nets are illustrated (some in more than one configuration) in Fig. 1.

Table 1. 4-connected plane nets

$\begin{array}{cccccccccc}m & \text { Not } & N & \varphi_{3} & \varphi_{4} & \varphi_{5} & \varphi_{6} & \varphi_{7} & \varphi_{8} & \varphi_{9} \\ 1 & 1 & 1 & - & 1 & - & - & - & - & - \\ 2 & 2 & 4 & \frac{1}{2} & - & \frac{1}{2} & - & - & - & - \\ 3 & 3 & 6,9 & \frac{1}{3} & \frac{1}{3} & \frac{1}{3} & - & - & - & - \\ & 4 & 3 & \frac{3}{3} & - & - & \frac{1}{3} & - & - & - \\ 4 & 5 & \mathbf{1 2} & \frac{3}{3} & - & - & - & \frac{1}{1} & - & - \\ & 6 & 4 & \frac{1}{2} & \frac{1}{4} & - & \frac{1}{4} & - & - & -\end{array}$

Table 2. Three-dimensional 4-connected nets Polygons in net

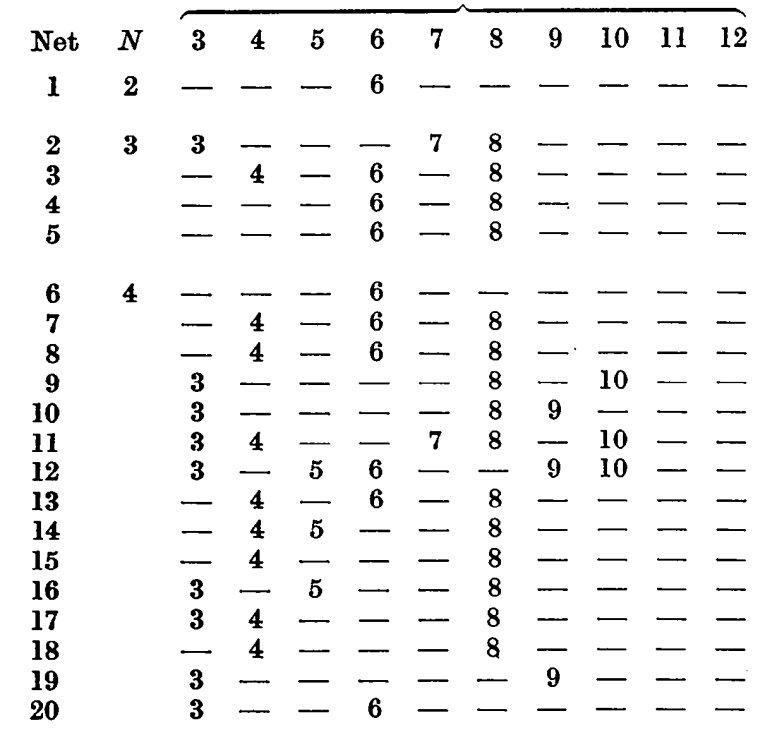

The three-dimensional nets will be listed in order of increasing numbers of points in the repeat unit (Table 2), but it is convenient to indicate their derivation under the headings $(a)-(d)$.

\section{(a) From 3-connected nets}

The simplest planar 3-connected net is the hexagonal net with 2 points in the repeat unit. By connecting alternate points to points in layers above and below (Fig. 2(a) and $(c)$ ) there arises the simplest threedimensional 4-connected net (Net 1) with 2 points in the (triclinic) unit cell. The highly symmetrical form of this net with 8 points in the cubic unit cell is the diamond structure.

The numbers of points in repeat units of plane 3 connected nets are even.

For 4 points in the repeat unit there are the possibilities:

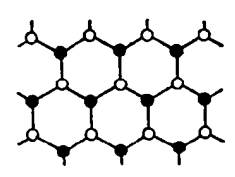

(a)

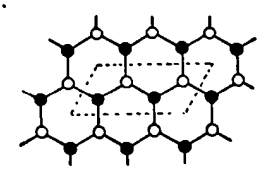

(e) Net 1

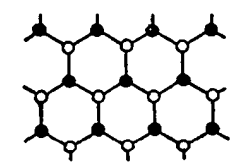

(b)

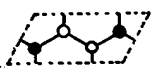

(f) Net 7

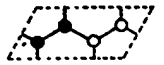

(g) Net 8

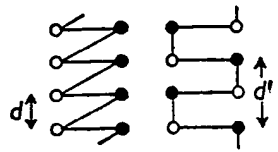

(c) Net 1 (d)Net 6

Fig. 2. Derivation of 4-connected nets (see text).

(1) Alternate layers $A$ (Fig. 2(a)) and its 'mirror image' $A^{\prime}$ (Fig. 2(b)) give the sequence of layers $A A^{\prime} A A^{\prime} \ldots$ shown in elevation in Fig. 2(d) with 4 points in the repeat unit (Net 6). For tetrahedral bonds this is the wurtzite structure. There is an indefinite number of more complex sequences of $A$ and $A^{\prime}$ layers; compare the $\mathrm{SiC}$ structures.

(2) In a double repeat unit of the plane hexagonal layer we may arrange two $D$ and two $U$ points as in Fig. $2(e),(f)$ and $(g)$. ( $D$ indicates a point to be connected downwards to a $U$ point of the layer below and $U$ a point to be connected upwards to a $D$ point of the layer above.) Of these the first is simply Net 1 ; the others are new nets, Nets 7 and 8.

(3) Planar 3-connected nets with 4 points in the repeat unit. No new nets arise from Nets 3 and 4 of Table 1 of Part 1.

For higher numbers of points in the repeat unit the three-dimensional nets can be derived in a similar way. The $A A^{\prime} A A^{\prime}$... type of net occurs only for nets with $4 n$ points in the repeat unit, since there are no planar 3-connected nets with odd numbers of points in the repeat units.

\section{(b) From mixed 3- and 4-connected nets}

In order to connect up to a three-dimensional 4connected net there must be an even number of 3connected points in the repeat unit. The simplest case is therefore the plane net with 3 points in the repeat unit. The nets arising here are shown in Fig. 3. They are:

3 points in unit cell: Nets 2 and 3 ;

4 points in unit cell: Nets 9-14.

(Fig. 3(c) does not give rise to a permissible net because $x=y$; see Part 1.)

\section{(c) From mixed 2- and 4-connected nets}

The plane nets arise by placing points on the lines of 4-connected nets. There is no plane 4-connected net with 2 points in the repeat unit (see Table 1).

2 points in repeat unit.-There is only one case: 14 -connected and 12 -connected point, and this is not permissible because $x=y$ (Fig. $4(a)$ ). 


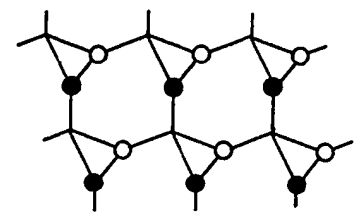

(a) Net 2

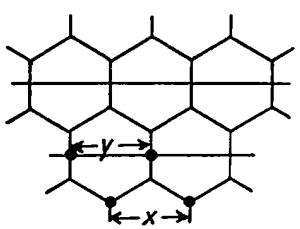

(c)

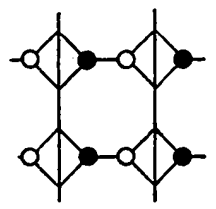

Net 9

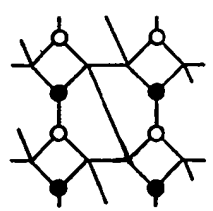

Net 14

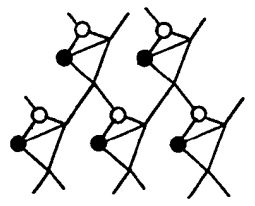

Net 10

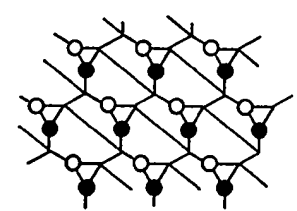

Net 12

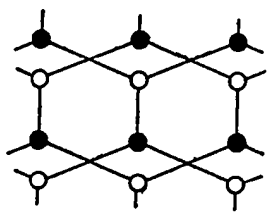

(b) Net 3

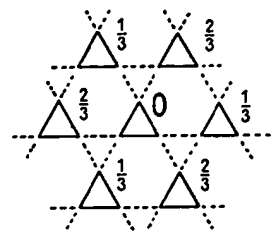

(d)

(e)

Fig. 3. (a) and (b) 4-connected Nets 2 and 3. (c) See text. (d) Projection of rhombohedral form of Net 2. (Heights as fractions of rhombohedral [111] axis.) (e) Derivation of 4-connected Nets 9-14.

3 points in repeat unit.-One net only: 14 -connected and 2 2-connected points. In Fig. $4(b)$ the point $A$ cannot be connected to corresponding points of layers above and below because this would make the distance between unconnected points $C C^{\prime}$ equal to $A A^{\prime}$ or $B B^{\prime}$, where primes indicate equivalent points in adjacent layers. There are, however, two other interesting possibilities involving the linking of point $A$ (Fig. 4(b)) of one layer to point $B^{\prime}$ of the layer above and point $D^{\prime}$ of either the layer below $(c)$ or of the layer above $(d)$. This mode of linking gives rise to screw axes, as shown at $(e)$ and $(f)$, where the numbers represent the heights of points above the plane of the paper in terms of $\frac{1}{3}$ (distance between layers). In (e) one-half of the triangles represent clockwise and the other half anti-clockwise helices This net (Net 4) can have rhombohedral or cubic symmetry. In $(f)$ all the triangles in the projection represent helices of the same sense (here anticlockwise); this is the enantiomorphic quartz type of net (Net 5). The most symmetrical forms of these nets are illustrated in Fig. 6 .
4 points in repeat unit.-No permissible nets arise.

\section{(d) From mixed 2-, 3- and 4-connected nets}

The relevant plane nets arise by placing (2-connected) points on the lines of the plane 3- and 4-connected nets of Fig. 3. Since there must be an even number of 3-connected points in the repeat unit the simplest nets will be formed from systems with 4 points in repeat unit: 12 -connected, 2 3-connected, and 1 4-connected. The permissible nets are set out in Fig. 5. Of the three ways of placing the 2-connected points in the third net only the third gives a permissible three-dimensional net.

We have now derived the 4-connected nets with 2,3 and 4 points in the repeat unit. They are summarized in Table 2, which lists the smallest polygons in the nets, and Nets 1-7 and 15 are illustrated, in their most symmetrical forms, in Fig. 6. Nets 1, 5, 6, 7 and 8 are illustrated as open packings of tetrahedra in Figs. 9-13. 


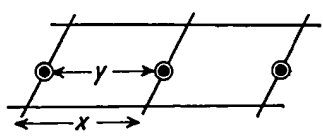

(a)

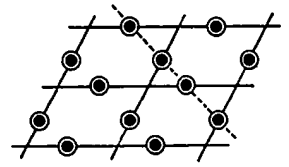

(c)

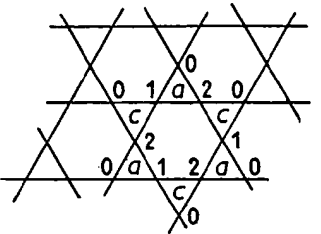

(e) Net 4
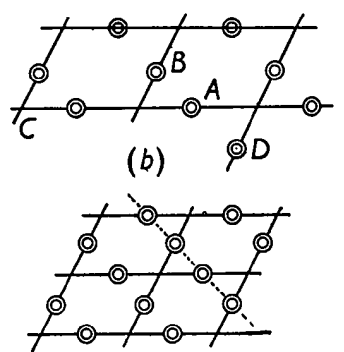

(d)

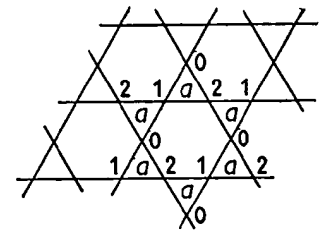

(f) Net 5
Point connected to points in layers above and below

(-) Point connected to points both in layer above or in layer helow

Fig. 4. Derivation of 4-connected nets (see text). In $(e)$ and $(f)$ heights are given in terms of one-third of the repeat distance between layers.

\section{Loops in 4-connected nets}

For 4-connected nets in which the same number of loops starts from each point there are two possibilities (Fig. 7): (a) two loops from each point, when only linear systems or closed rings are possible; and $(b)$ one loop from each point. The nets arising under $(b)$ are exactly analogous to those for 3-connected points, being 3-connected nets in which one-third of the links have been replaced by loops. There are 4-connected nets of this kind corresponding to each of the threedimensional 3-connected nets.

If we drop the condition that the same number of loops starts from each point then series of nets can be derived containing points of type $(a)$ and/or $(b)$. Any number of points of type $(a)$ may be added between pairs̀ of points already connected by a loop, as at $(c)$, but it is doubtful whether such nets are of interest in the present connection.

The simplest three-dimensional 4-connected net containing loops is of interest in connection with the systematic derivation of 3-connected from 4-connected nets by replacing<smiles>CC(C)C(C)C(C)C</smiles>

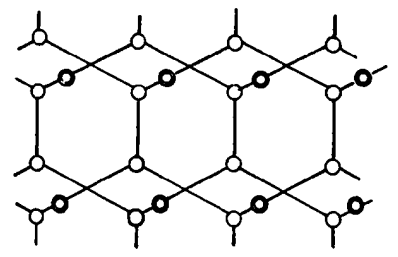

Net 15

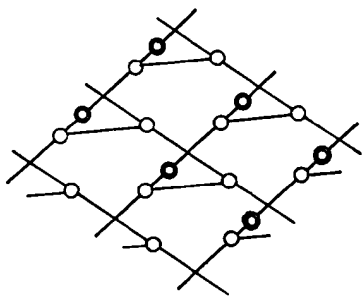

Net 17

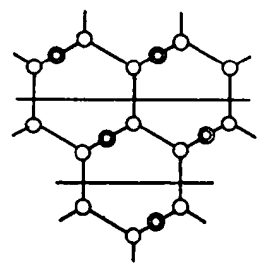

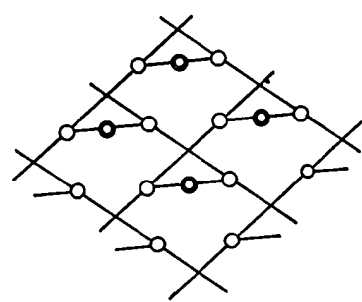

Net 18

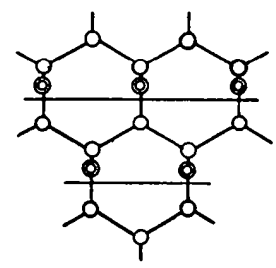

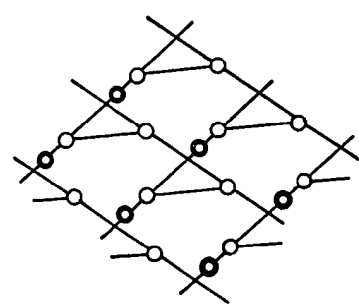

Net 19

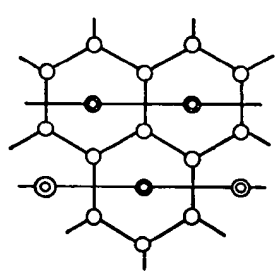

Net 20

Fig. 5. Derivation of 4-connected Nets 15-20. 


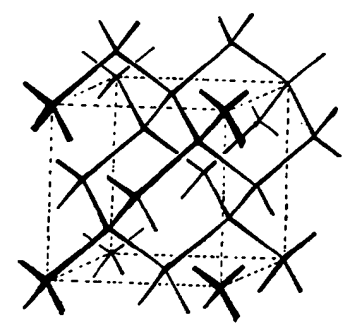

1
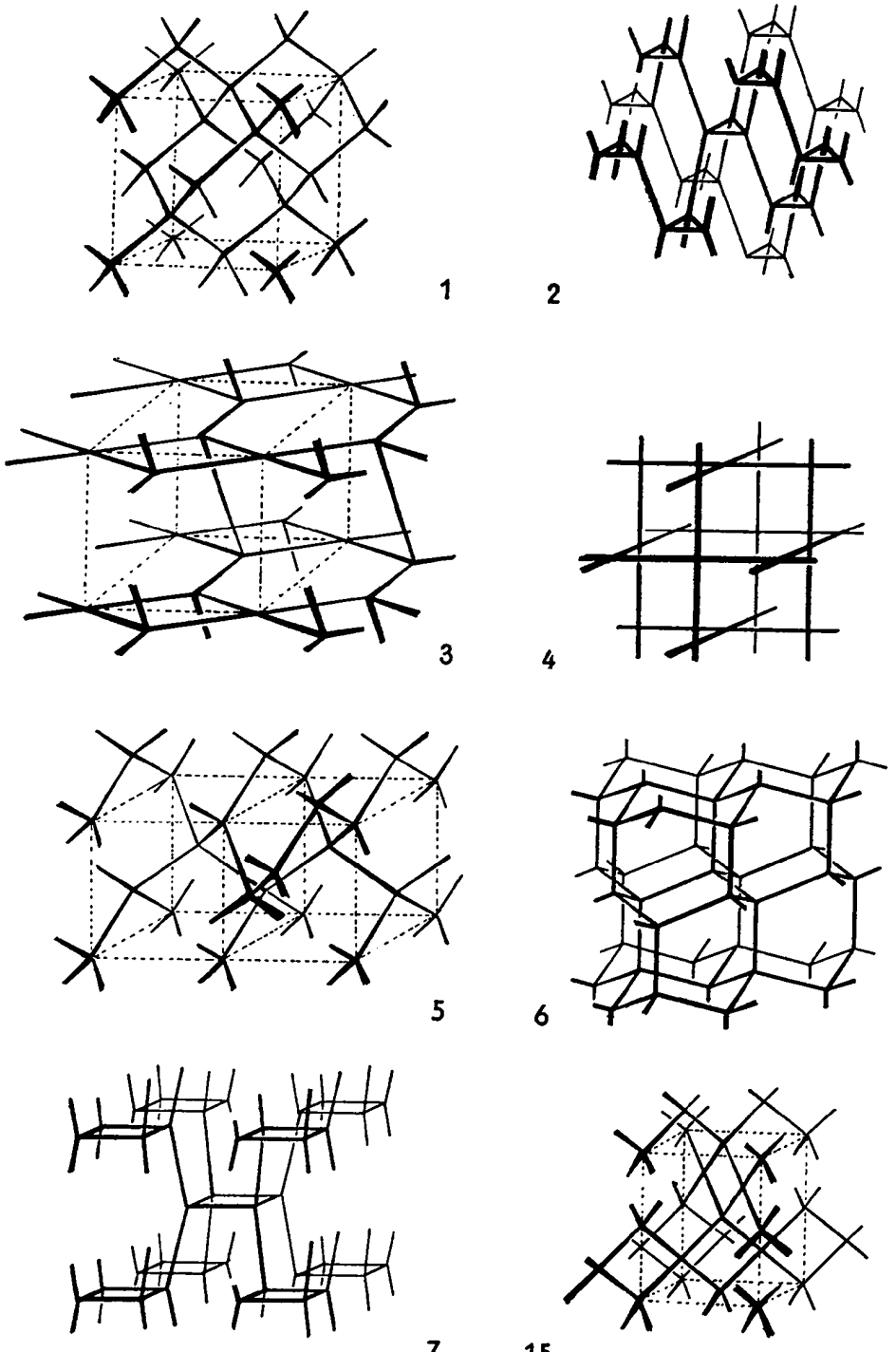

Fig. 6. Some three-dimensional 4-connected nets. The numbering is that of Table 2.

thereby doubling the number of points in the unit cell. In this way the 4-connected Net 1 gives the 3 -connected Nets 1 or 2 depending on the relative orientations of the 2-point units. The nineteen three-dimensional 3-con-

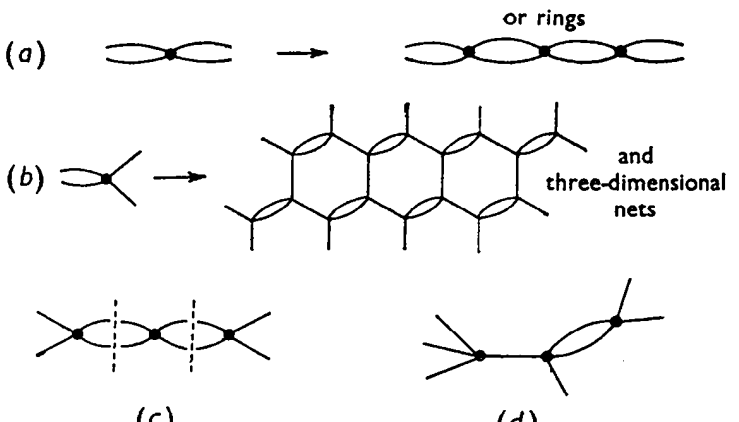

(c)

(d)

Fig. 7. Loops in 4-connected nets. nected nets with 6 points in the unit cell must be derivable from the 4-connected nets with 3 points in the unit cell. It can easily be verified that the 3 -connected Net 15 cannot be derived in this way from any of the 4-connected Nets 2-5 of Table 2. This net (and also the 3-connected Net 14) can, however, be obtained in this way from the simplest 4-connected net containing loops, namely, that based on the 3-point repeat unit $(d)$ of Fig. 7. This new 4-connected net is illustrated in Fig. 8, which also shows the 3-connected Nets 14 and 15 drawn in similar orientations.

Nets containing loops are important because by placing points along the links they represent structures of compounds $A X_{2}, A X_{3}$, etc. formed by the sharing of edges of planar, tetrahedral, octahedral, etc. coordination groups. Thus the linear system of Fig. 7(a) represents in this way the structures of crystalline $\mathrm{CuCl}_{2}$ (or $\mathrm{CuBr}_{2}$ ) and $\mathrm{SiS}_{2}$ : 


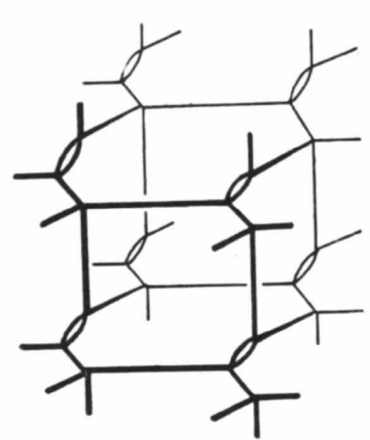

(a)

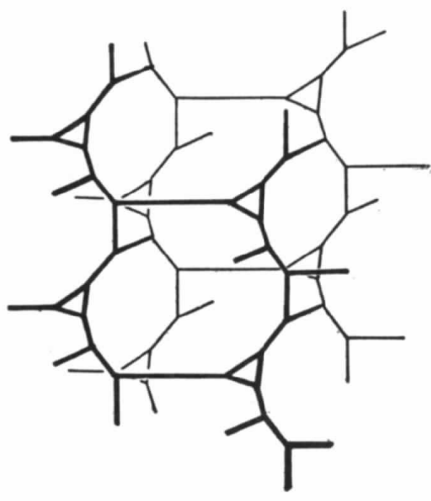

(b) Net 14

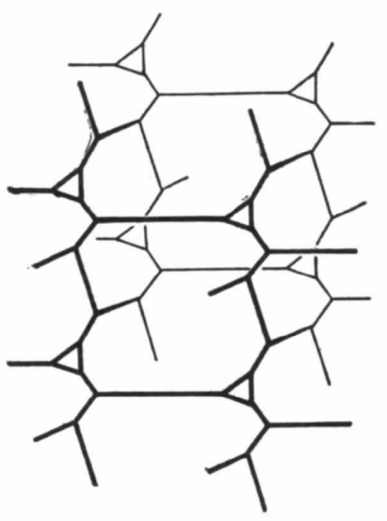

(c) Net 15

Fig. 8. Relation between the simplest three-dimensional 4-connected net containing loops (a), and the 3-connected Nets 14 and 15.
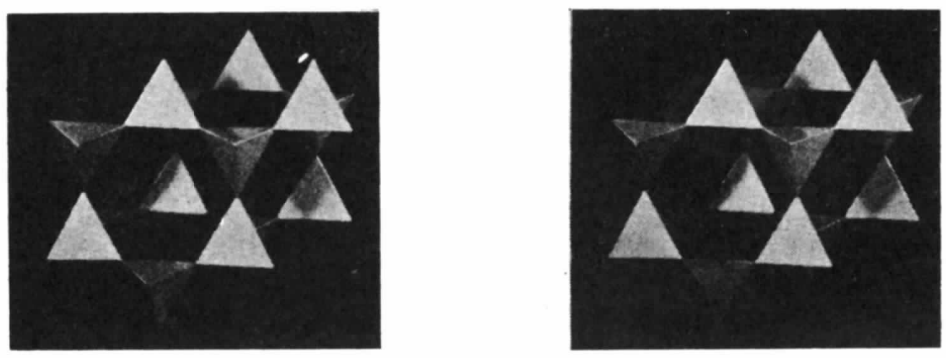

Fig. 9.
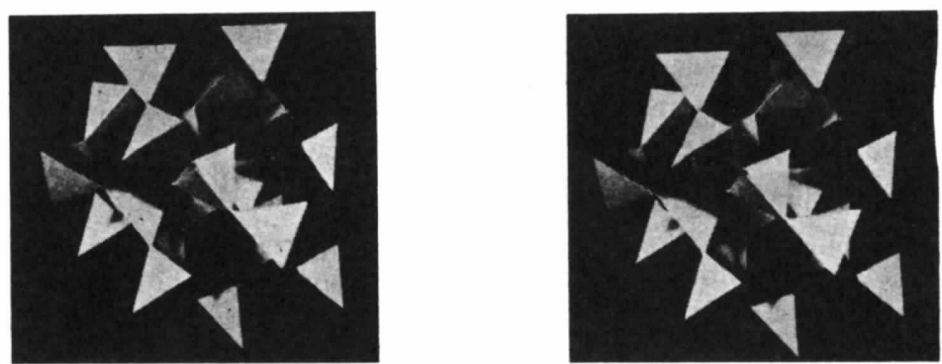

Fig. 10.
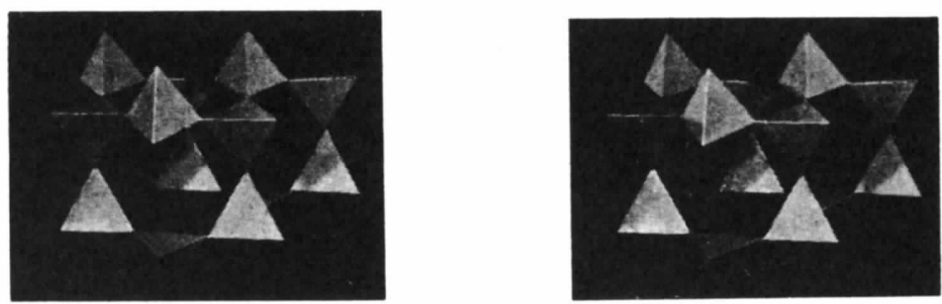

Fig. 11 . 

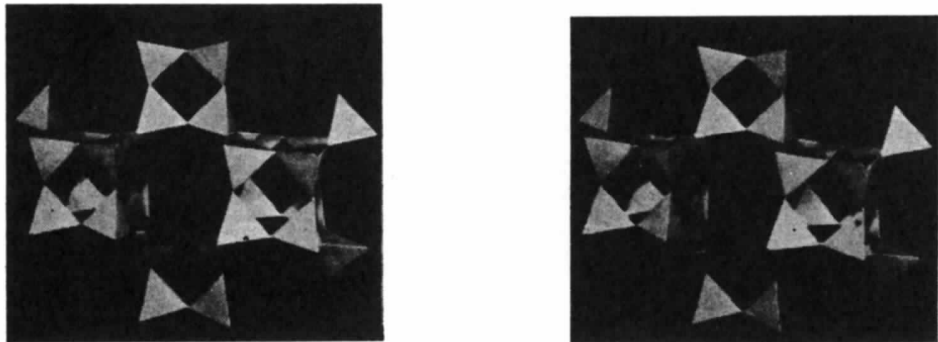

Fig. 12.
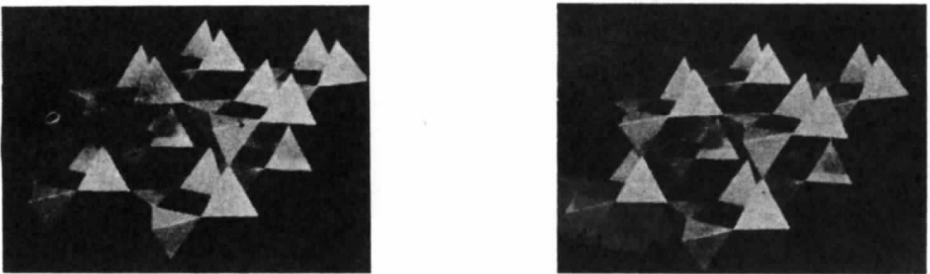

Fig. 13.

$$
>_{A}\left\langle\begin{array}{l}
X \\
{ }_{X}
\end{array}\right\rangle_{A}\left\langle\begin{array}{l}
X \\
{ }_{X}
\end{array}\right\rangle_{A}\left\langle\begin{array}{l}
X \\
{ }_{X}
\end{array}{ }_{A}\left\langle\begin{array}{l}
X \\
{ }_{X}
\end{array}\right\rangle_{A}\langle\right.
$$

The corresponding structures based on, for example, tetrahedra sharing all corners are obtained simply by placing an $X$ atom along each link of certain of the 4-connected nets of Table 2. These two types of net therefore provide a way of studying the most regular types of open packings of polyhedra.

\section{Open packings of tetrahedra}

Space-filling by polyhedra, i.e. the close-packing of polyhedra, received attention many years ago, outstanding contributions being the enumeration by Fedorov (1904) of the five types of polyhedra which fill space when packed together in the same orientation and the work of Andreini (1907) on space-filling combinations of regular and semi-regular solids, to which we refer later. Few systematic studies seem to have been made of open packings of polyhedra, though they are of great interest in crystallography.

Polyhedra may be placed together so that faces, edges, or vertices, or combinations of these, are in contact. Arrangements of tetrahedra in which each is in contact with four others at its vertices follow directly from the 4-connected nets of which the simplest were listed in Table 2. Certain of these nets can be realised with equal links and a regular tetrahedral arrangement of links at each point. The lines joining the mid-points of the links meeting at each point are the edges of regular tetrahedra described around the points of the original net. Each tetrahedron is in contact with four others at its vertices (the mid-points of the links of the 4-connected net) and the system of linked tetrahedra is necessarily periodic in three dimensions. If the tetrahedra represent $\mathrm{SiO}_{4}\left(\right.$ or $\left.\mathrm{AlO}_{4}\right)$ groups the tetrahedron packings represent the idealized structures of the forms of silica and of aluminosilicates with framework structures.

Corresponding to the nets of Table 2 there are the following tetrahedron packings:

$$
\begin{array}{cc}
\text { Net 1: cristobalite structure } & \text { Fig. 9 } \\
\text { 5: quartz structure } & \text { Fig. 10 } \\
\text { 6: tridymite structure } & \text { Fig. 11 } \\
\text { 7: } & \text { Fig. 12 } \\
\text { 8: } & \text { Fig. 13 }
\end{array}
$$
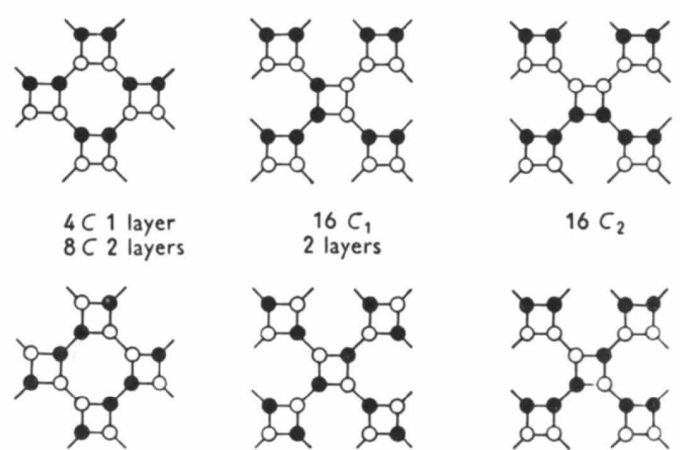

$16 C_{1}$

2 layers

$16 C_{2}$

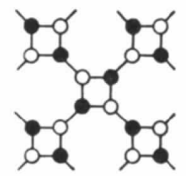

$8 T_{1}$

2 layers

$8 T_{2}$
1 layer

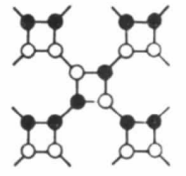

$16 C T$

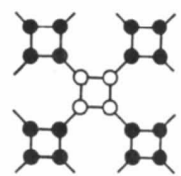

$8 U$

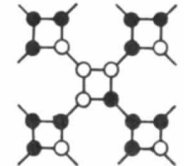

$8 V$

Fig. 14. Derivation of open packings of tetrahedra. 

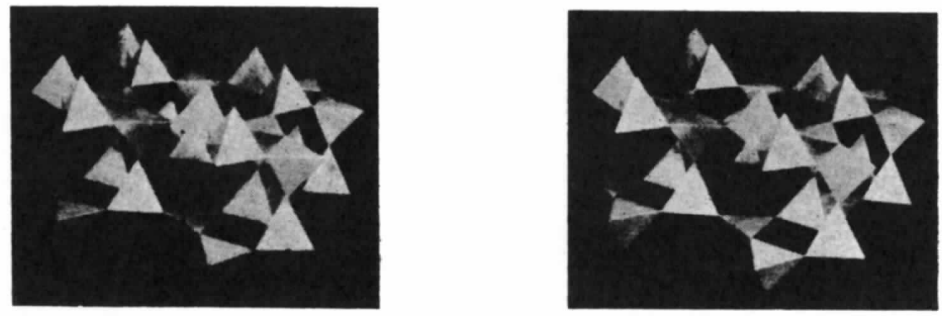

Fig. 15.
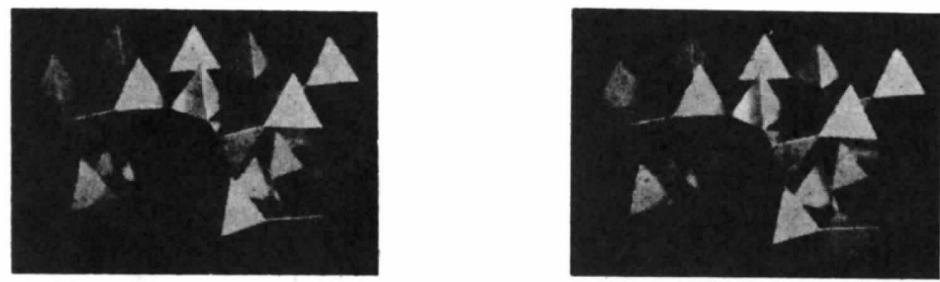

Fig. 16.
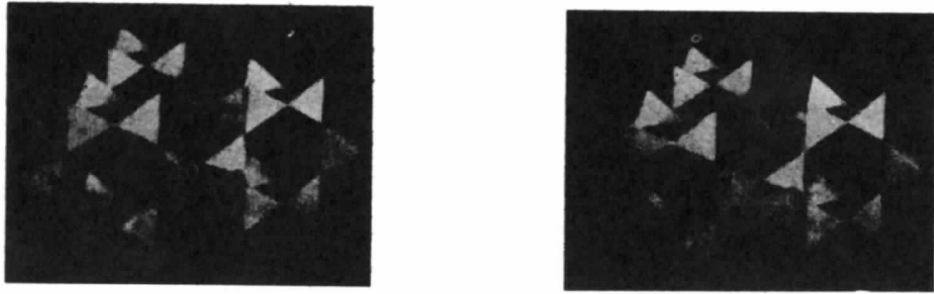

Fig. 17.

The structures adopted by silica represent the three simplest ways of joining up tetrahedral groups in three dimensions. It is therefore of interest to enquire whether a particular framework silicate adopts the simplest of a family of related structures. This point may be illustrated by reference to the felspars.

The structures of Figs. 9 and 11 are built from layers in which tetrahedra are arranged at the points of the simplest plane 3-connected net (the hexagonal net), the unattached vertices pointing alternately up and down. Such a layer may be represented diagrammatically as in Fig. 2(a), each tetrahedron being linked to three others in the plane and having its fourth vertex pointing downwards (black circle) or upwards (open circle). In Fig. 14 are shown the simpler possibilities for the plane $4: 8$ net. If the pattern of black circles in a layer is the same as that of the open circles then in order that the layers may be joined together it is only necessary to translate each one relative to the adjacent layers, as in Fig. 13. If this is not the case, the three-dimensional structure must be built of pairs of layers related by planes of symmetry. In Fig. 14 $C$ and $T$ indicate whether adjacent or opposite tetrahedra in a 4-ring point in the same direction (upwards or downwards).

It is interesting to note that the idealized felspar structure (Taylor, 1933) is the rather complex packing

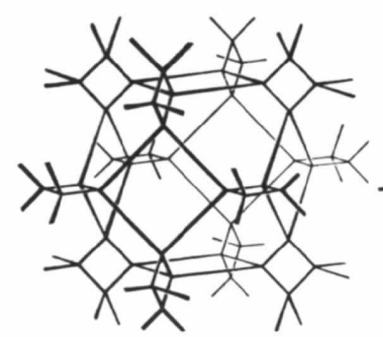

(a)

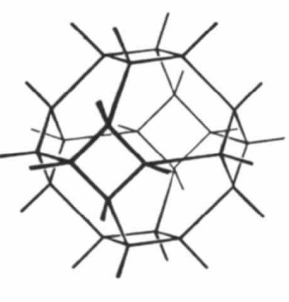

(b)
Fig. 18. Configurations of the 4-connected net $(a)$ of Table 3 .

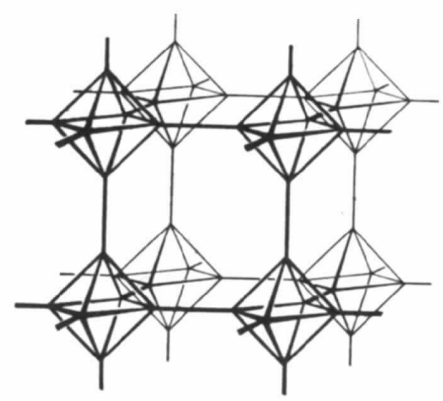

(a)

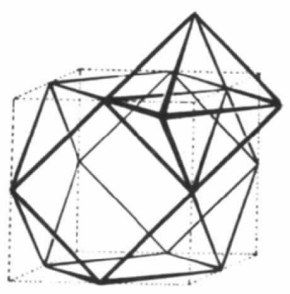

(b)
Fig. 19. The cubic nets $(d)$ and $(f)$ of Table 3. 
Table 3

\begin{tabular}{|c|c|c|c|c|c|}
\hline Net & $p$ & Space-group & Equivalent position & Coordinates & Remarks \\
\hline $\begin{array}{l}(a) \\
(b)\end{array}$ & 4 & $\begin{array}{l}\text { Pn } 3 m \\
F d 3 m \\
I 4_{1} 3 \\
I m 3 m \\
I a 3 d\end{array}$ & $\begin{array}{r}6(d) \\
8(a) \\
12(c) \\
12(d) \\
24(c)\end{array}$ & $\begin{array}{l}- \\
- \\
-\end{array}$ & $\begin{array}{l}\text { Net } 4 \text { Fig. } 6 \\
\text { Net } 1 \text { Fig. } 6 \text { (H. \& L. } 4_{1} \text { ) } \\
\text { H. \& L. } 4_{3} \\
\text { Andreini Fig. } 14 \text { (ot) }\end{array}$ \\
\hline & & $P m 3 m$ & $24(k)$ & $\begin{array}{l}y=0.369 \\
z=0 \cdot 185\end{array}$ & Andreini Fig. $21(c, o t, c o t)$ \\
\hline (c) & & $\begin{array}{l}F d 3 m \\
P m 3 m\end{array}$ & $\begin{array}{l}32(e)^{*} \\
48(n)\end{array}$ & $\begin{array}{l}x=0.069 \\
x=0.256 \\
y=0 \cdot 104 \\
z=0.397\end{array}$ & $\begin{array}{l}\text { H. \& I. } 4_{4} \\
\text { Andreini Fig. } 24^{\prime}\left(p_{8}, c o t\right)\end{array}$ \\
\hline & & $F m 3 m$ & $96(k)$ & $\begin{array}{l}x=0.193 \\
z=0.079\end{array}$ & $\begin{array}{l}\text { Andreini Fig. } 23(t t, c t, c o t) \\
\text { H. \& L. } 4_{2}\end{array}$ \\
\hline (d) & 5 & $\begin{array}{l}P m 3 m \\
P m 3 m\end{array}$ & $\begin{array}{c}6(e) \\
24(m)\end{array}$ & $\begin{array}{l}x=0.293 \\
x=0 \cdot 138 \\
z=0 \cdot 362\end{array}$ & $\begin{array}{l}\text { Andreini Fig. } 17(o, c t) \\
\text { Andreini Fig. } 22\left(c, p_{8}, c t, r c o\right)\end{array}$ \\
\hline & & $F m 3 m$ & $96(j)$ & $y=\frac{1}{8}, z=\frac{1}{6}$ & Andreini Fig. $24(t t, c o, o t)$ \\
\hline (e) & 6 & $\begin{array}{l}P \text { lattice } \\
P 4_{1} 3 \\
F d 3 m\end{array}$ & $\begin{array}{r}\overline{4(a)} \\
16(c)\end{array}$ & - & $\begin{array}{l}\text { Andreini Fig. } 5(c) \\
\text { Andreini Fig. } 15(t, t)\end{array}$ \\
\hline & & $\begin{array}{l}\operatorname{Pm} 3 m \\
F m 3 m\end{array}$ & $\begin{array}{l}12(j) \\
96(k)\end{array}$ & $\begin{array}{l}x=0 \cdot 207 \\
x=0 \cdot 146 \\
z=0 \cdot 354\end{array}$ & $\begin{array}{l}\text { Andreini Fig. } 20(c, c o, r c o) \\
\text { Andreini Fig. } 19(t, c, r c o)\end{array}$ \\
\hline$(f)$ & 8 & $\begin{array}{l}I \text { lattice } \\
P m \mathbf{3} m \\
I \overline{\mathbf{4}} \mathbf{3} d\end{array}$ & $\begin{array}{r}\overline{3(c)} \\
12(a)\end{array}$ & - & Andreini Fig. $18(o, c o)$ \\
\hline & 12 & $F$ lattice & - & - & Andreini Fig. $12(t, o)$ \\
\hline
\end{tabular}

Notes:

(a). The links in this net outline truncated octahedra (Fedorov's fifth space-filling parallelepiped). A less symmetrical variant of this net (e.g. the position $12(h)$ in $\operatorname{Pm} 3 m, x \neq 1$ ) is shown in Fig. 18(a); for $x=\frac{1}{4}$ it becomes the net of Fig. 18(b). This net is the basis of the ultramarine structure.

$(b)$. The net $I 4_{1} 312(c)$ is enantiomorphic. The net $(b)$ consists of two interpenetrating but independent nets of this kind, one l. and the other $r$-handed. It may be described as a three-dimensional racemate. The net $I a 3 d$, $16(b)$, bears exactly the same relationship to the 3-connected Net $1\left(I 4_{1} 3,8(a)\right)$, being also a three-dimensional racemate.

$(c)$ and $(e)$. Tetrahedra may be placed at the points of the diamond net to give a 4-connected net $(c)$ with discrete tetrahedra, two points being placer along each link, or a 6-connected net (e) by joining up mid-points of adjacent links. Of these (c) corresponds to the open-packing $\mathbf{4}_{4}$ of Heesch \& Laves, and $(e)$ to Andreini's packing of equal numbers of tetrahedra and truncated tetrahedra.

$(d)$ and $(f)$. Octahedra may be placed at the points of a cubic lattice to give the 5-connected net $(d)$ of Fig. 19(a), which is Andreini's packing of octahedra and truncated cubes, or the 8-connected net $(f)$ of Fig. 19(b), which is a packing of equal numbers of octahedra and cuboctahedra. The net $(d)$ represents the boron framework in the $\mathrm{CaB}_{6}$ structure.

$16 C_{1}$, whereas that of paracelsian (Smith, 1953) is the simpler $8 C$ structure of Fig. 15. (Owing to the distortion of the layers the actual unit cell contains $16 \mathrm{Si}(\mathrm{Al}) \mathrm{O}_{4}$ tetrahedra like the felspars.) The structures of Figs. 15 and 13 are related in the same way as those of Figs. 11 and 9 . The $4 C$ and $8 T$ structures of Figs. 13 and 16 do not appear to be known. Fig. 17 illustrates the open sphere packing $4_{2}$ of Heesch \& Laves (1933) as a packing of tetrahedra; it is the trigonal analogue of the packing of Fig. 12.

\section{Some frameworks with cubic symmetry}

In Table 4 of Part 1 we listed some 3-connected nets with cubic symmetry which are realizable with equal links and equivalent points. In the course of this work a list has been made of more highly connected networks of this type, some of which are of interest in structural problems (Table 3). In certain of these nets the links outline polyhedra. Andreini (1907) considered a special case of space-filling, namely, by regular or semi-regular polyhedra, alone or in combination. Table 3 includes all Andreini's space-fillings which have cubic symmetry; the numbers of the figures in Andreini's paper are given, and his symbols are retained: tetrahedron $(t)$, truncated tetrahedron $(t t)$, cube $(c)$, truncated cube $(c t)$, octahedron $(o)$, truncated octahedron (ot), cuboctahedron (co), truncated cuboctahedron (cot), rhombi-cuboctahedron ( $r c o)$, octagonal prism $\left(p_{8}\right)$.

Table 3 also includes the cubic forms of the nets corresponding to the sphere packings $4_{1}-4_{4}$ of Heesch 
\& Laves. Of these $4_{1}$ is the diamond net, $4_{3}$ arises by joining up the mid-points of adjacent links in $\mathbf{3}_{\mathbf{1}}$ (see Part 1), and $\mathbf{4}_{4}$ results from placing a tetrahedral group at each point of $4_{1}$. The hexagonal form of $4_{1}$ is the 4-connected Net 6 of Table 2 and Figs. 2 and 11; the hexagonal variant of $\mathbf{4}_{2}$ has been illustrated in Fig. 17 as a packing of tetrahedra.

\section{Inert gas hydrate structures}

To complete our discussion of 4-connected nets we mention here the structures which have been proposed (Claussen, $1951 a, b, c$ ) for hydrates with formulae close to $X .6 \mathrm{H}_{2} \mathrm{O}$ and $Y .17 \mathrm{H}_{2} \mathrm{O}$ where $X$ is $\mathrm{A}, \mathrm{CH}_{4}, \mathrm{CO}_{2}$, etc. and $Y$ is $\mathrm{CH}_{3} \mathrm{I}, \mathrm{C}_{2} \mathrm{H}_{5} \mathrm{Cl}$, etc. These structures are open packings of water molecules with the inert gas or other material in the interstices. Since each water molecule in an 'expanded ice' framework has four neighbours arranged tetrahedrally the required networks are 4-connected. It might have been expected that the nets would have been found among those in which the links outline polyhedra in the spacefillings of Andreini (Table 3). Instead, these hydrate structures correspond to very elegant space-fillings by (a) pentagonal dodecahedra and 14-hedra (with 12 5 -gon and 2 6-gon faces), and (b) pentagonal dodecahedra and 16-hedra (with 12 5-gon and 4 6-gon faces). The unit cells contain respectively 46 and 136 water molecules.

\section{References}

ANDrein,, A. (1907). Mem. Soc. ital. Sci. nat. (3), 14, 75. Cladussen, W. F. (1951a). J. Chem. Phys. 19, 259. Chaussen, W. F. (1951b). J. Chem. Phys. 19, 662. Cluadssen, W. F. (195lc). J. Chem. Phys. 19, 1425. Fedorov, E. v. (1904). Z. Kristallogr. 38, 321. HefsCh, H. \& Laves, F. (1933). Z. Kristallogr. 85, 443. Smith, J. V. (1953). Acta Cryst. 6, 613.

TAYLOR, W. H. (1933). Z. Kristallogr. 85, 425. WerLs, A. F. (1954). Acta Cryst. 7, 535.

Acta Cryst. (1954). 7, 554

\title{
An X-ray Microbeam Study of Polycrystalline Specimens of Aluminium and Iron Deformed in Tension
}

\author{
BY A. KrLLY* \\ Crystallographic Laboratory, Cavendish Laboratory, Cambridge, England
}

(Received 11 March 1954)

\begin{abstract}
Polycrystalline specimens of pure aluminium and of iron have been examined with the X-ray microbeam technique at various stages of a tensile test. From the $X$-ray photographs, measurements have been made of the size of the particles formed within the grains during deformation, of the breadths of the X-ray reflexions from these crystallites and of the mean angle between them. The particles are identified with the regions between slip bands in the material and an attempt is made to account for the stress-strain curve of the specimens from this hypothesis and the results of a dislocation theory due to Frank. Reasonable agreement is found between calculation and experiment.
\end{abstract}

\section{Introduction}

$\mathrm{X}$-ray microbeam methods have been used previously to examine a number of polycrystalline metals after deformation by rolling (Hirsch \& Kellar, 1952; Hirsch, $1952 a, b ;$ Gay \& Kelly, 1953a, b). It was decided to carry out an investigation of specimens of aluminium and iron deformed in tension, first, because the specimens are then in a more precisely defined mechanical state, and second, to test whether a different method of deformation resulted in the development of a different texture within the material.

The interpretation of the earlier results has led to

* Now at Department of Mining and Metallurgical En. gineering, University of Illinois, Urbana, U.S.A. the formulation of a theory to account for the ultimate yield stress of polycrystalline metals containing a particle structure of the type found (Gay, Hirsch \& Kelly, 1954). The results presented in this paper are used in an attempt to extend this theory to explain the work-hardening of the materials in terms of the experimentally observed variation of particle size with plastic strain.

Polycrystalline aluminium has been thoroughly investigated because this material is particularly suitable for examination with the X-ray microbeam technique. Fewer experimental results are available for iron specimens but these are of importance because they confirm the existence of particles of size $\sim 1-2 \mu$ found within the rolled material (Gay \& Kelly, 1953a). 